CALT-68-2326

hep-ph/0104169

\title{
Supersymmetric Correction to Top Quark Pair Production near Threshold
}

\author{
Shufang Su* and Mark B. WiSE ${ }^{\dagger}$ \\ California Institute of Technology, Pasadena, California 91125
}

\begin{abstract}
We studied the leading supersymmetric contribution to top-antitop threshold production using the NRQCD framework. The one-loop matching to the potential and the Wilson coefficient of the leading ${ }^{3} S_{1}$ production current were considered. We point out that the leading correction to the potential is zero due to $\mathrm{SU}(3)_{c}$ gauge invariance. This is true in general for any new physics that enters above the electroweak scale. The shape of the top quark pair production cross section is therefore almost unaffected near threshold, allowing a precise determination of the top quark mass based on the Standard Model calculations. The supersymmetric correction to the Wilson coefficient $c_{1}$ of the production current decouples for heavy super particles. Its contribution is smaller than the Standard Model next-to-next-leading-log results.
\end{abstract}

PACS numbers:14.65.Ha, 12.60.Jv, 12.38.Bx.

*shufang@theory.caltech.edu

${ }^{\dagger}$ wise@theory.caltech.edu 
It is likely that top quark pair production near threshold will be investigated intensively in the future at an $e^{+} e^{-}$linear collider. It can be used to determine the top quark mass, decay width, top Yukawa coupling and strong coupling constant. The Standard Model (SM) QCD prediction has been studied in great detail by several groups [1,2] in the framework of NRQCD (nonrelativistic QCD) [3] up to next-to-next-leading-log (NNLL) order. Near the threshold, the velocity of the top quark $v \sim \alpha_{s} \sim 0.15$ is small. However, the relevant energy scales $m_{t} \sim 175 \mathrm{GeV}, m_{t} v \sim 25 \mathrm{GeV}, m_{t} v^{2} \sim 4 \mathrm{GeV}$, together with top decay width $\Gamma_{t} \approx 1.5$ $\mathrm{GeV}$ are much larger than $\Lambda_{\mathrm{QCD}}$. Therefore, top quark production can be calculated to high precision using perturbative methods.

Schematically, the $t \bar{t}$ cross section $\sigma_{t \bar{t}}$ can be expressed as a series:

$$
\sigma_{t \bar{t}} \sim v \sum_{k, i}\left(\frac{\alpha_{s}}{v}\right)^{k}\left(\alpha_{s} \ln v\right)^{i}\left\{\begin{array}{ll}
1 & (\mathrm{LL}) \\
\alpha_{s}, v & (\mathrm{NLL}) \\
\alpha_{s}^{2}, \alpha_{s} v, v^{2} & (\mathrm{NNLL})
\end{array} .\right.
$$

The velocity renormalization group (VRG) [4] summation of $\alpha_{s} \ln v$ and Coulomb summation of $\alpha_{s} / v$ are performed to all orders. SM QCD calculations have been performed at NNLL order [2], and the remaining theoretical uncertainties are likely to be at the few percent level.

Supersymmetry (SUSY) is the most prominent extension of the SM, and gives new contributions to top quark production through loop corrections. Supersymmetric one-loop corrections to the process $e^{+} e^{-} \rightarrow t \bar{t}$ above the threshold region have been studied in [5]. For scales below $m_{t}$, heavy super particles decouple and they only affect $t \bar{t}$ pair production in the threshold region through matching conditions at the scale $\mu=m_{t}$. For heavy super particles, the supersymmetric contribution is of the order of $\sigma_{t \bar{t}} \sim v \alpha_{s}\left(m_{t} / M_{\mathrm{SUSY}}\right)^{n}$, where $M_{\mathrm{SUSY}}$ is the scale of the super particle masses, which is smaller than $1 \mathrm{TeV}$ if supersymmetry plays a role in explaining the hierarchy puzzle. Therefore, the leading SUSY correction could be comparable to the SM NNLL corrections. In this paper, we will study the supersymmetric contribution to top pair threshold production through one-loop matching conditions, neglecting subleading corrections which are suppressed by higher powers of $\alpha_{s}$ or $v$. We do not consider SUSY loop corrections to the width of the top. They were considered in [6].

For $t \bar{t}$ production in $e^{+} e^{-}$annihilation via a photon or $Z$ boson exchange, the normalized cross section in the full QCD theory (at c.m. energy $\sqrt{s}$ ) is related to

$$
\operatorname{Im}\left[-i \int d^{4} x e^{i q \cdot x}\left\langle 0\left|T \boldsymbol{J}(x) \boldsymbol{J}^{\dagger}(0)\right| 0\right\rangle\right],
$$

where $q=(\sqrt{s}, 0)$ and $\boldsymbol{J}$ is the production current. In NRQCD, The non-relativistic ${ }^{3} S_{1}$ current for top quark production near threshold can be written as [2]

$$
\boldsymbol{J}=c_{1} \boldsymbol{O}_{\boldsymbol{p}_{, 1}}+\ldots,
$$

where $\boldsymbol{O}_{\boldsymbol{p}_{1}}=\psi^{\dagger} \boldsymbol{p} \boldsymbol{\sigma}\left(i \sigma_{2}\right) \chi_{-\boldsymbol{p}}^{*}$ and the ellipses denote terms suppressed by powers of $v$. The correlator of the currents $\boldsymbol{J}$ in Eq. (21) is replaced by the correlator of $\boldsymbol{O}_{p_{1,1}}$, which is proportional to the coordinate space Green function $G(0,0)$, with its momentum space Fourier transformation $\tilde{G}\left(\boldsymbol{p}, \boldsymbol{p}^{\prime}\right)$ being a solution of the Schrödinger equation [2]: 


$$
\left[\frac{\boldsymbol{p}^{2}}{m}-E-i \Gamma_{t}\right] \tilde{G}\left(\boldsymbol{p}, \boldsymbol{p}^{\prime}\right)+\int D^{n} \boldsymbol{q} \mu_{S}^{2 \epsilon} \tilde{V}(\boldsymbol{p}, \boldsymbol{q}) \tilde{G}\left(\boldsymbol{q}, \boldsymbol{p}^{\prime}\right)=(2 \pi)^{n} \delta^{(n)}\left(\boldsymbol{p}-\boldsymbol{p}^{\prime}\right),
$$

where $m$ is the heavy quark pole mass, $E \equiv(\sqrt{s}-2 m), n \equiv 3-2 \epsilon$ and $D^{n} \boldsymbol{q} \equiv$ $e^{\epsilon \gamma_{E}}(4 \pi)^{-\epsilon} d^{n} \boldsymbol{q} /(2 \pi)^{n}$. $\tilde{V}(\boldsymbol{p}, \boldsymbol{q})$ is the color singlet quark-antiquark potential in NRQCD, with the leading term being just the Coulomb potential. Therefore, the cross section for $t \bar{t}$ production near threshold is proportional to the product of the coefficient $c_{1}$ with the associated Green function.

The main ingredients in the calculation of the top pair production cross section using NRQCD are the potential $\tilde{V}(\boldsymbol{p}, \boldsymbol{q})$ for a quark-antiquark pair and the Wilson coefficient $c_{1}$ of the production current, which are obtained via matching to the coefficients of the relevant operators calculated at the scale $\mu=m_{t}$, then running down to lower scales using the VRG [4]. Recent SM calculations at NNLL order [7,8] are likely to have reduced the theoretical uncertainties to a few percent level. In what follows, we will consider the SUSY contribution to both the potential for a quark-antiquark pair and the Wilson coefficient $c_{1}$ of the ${ }^{3} S_{1}$ current, through one-loop matching conditions.

The potential interaction in NRQCD is written as

$$
\mathcal{L}=-V_{\alpha \beta \lambda \tau}\left(\boldsymbol{p}, \boldsymbol{p}^{\prime}\right) \psi^{\dagger} \boldsymbol{p}_{\alpha}^{\prime} \psi_{\boldsymbol{p}_{\beta}} \chi^{\dagger}-\boldsymbol{p}^{\prime}{ }_{\lambda} \chi-\boldsymbol{p}_{\tau},
$$

where $\alpha, \beta, \lambda, \tau$ are the color and spin indices. $\psi_{\boldsymbol{p}}$ and $\chi_{\boldsymbol{p}}$ are in the $\mathrm{SU}(3)_{c}$ representation of $\mathbf{3}$ and $\overline{\mathbf{3}}$, which annihilate a quark and an antiquark, respectively. The leading term in $V$ is 8 :

$$
V=\left(T^{A} \otimes \bar{T}^{A}\right) \frac{V_{c}^{(T)}}{\boldsymbol{k}^{2}},
$$

where $\boldsymbol{k}=\boldsymbol{p}^{\prime}-\boldsymbol{p}$. At leading order, the SUSY contribution to the Coulomb term $V_{c}^{(T)}$ is always zero by $\mathrm{SU}(3)_{c}$ gauge invariance. Integrating out heavy supersymmetric particles generates gauge invariant local operators at the top quark scale. In the transition to NRQCD at leading order in $\alpha_{s}$, only the kinetic term for the top quark generates the Coulomb potential. The subleading terms in the potential could get contributions from supersymmetric loops. However, they are suppressed by powers of $v$ relative to the leading Coulomb potential.

For more general types of new physics that enter above the electroweak scale, and contribute to top quark pair production via perturbative matching at $\mu=m_{t}$, the Coulomb potential is also protected due to the requirement of $\mathrm{SU}(3)_{c}$ gauge invariance. Therefore, the shape of the top pair production cross section, which is related only to the Green function in Eq. (4), will stay almost unchanged, allowing a precise determination of the top quark mass based solely on the SM calculations, with uncertainties that may be as small as 100 $\mathrm{MeV}$ [1].

The Feynman diagrams that contribute to the leading Wilson coefficient $c_{1}$ of the ${ }^{3} S_{1}$ current are shown in Fig. 1. The SUSY corrections $\delta c_{1 \gamma}$ or $\delta c_{1 Z}$ to the production current associated with a photon or $Z$ boson are defined as:

$$
\begin{aligned}
\text { photon coupling : } & \boldsymbol{A}\left(\frac{2}{3} e\right) \boldsymbol{O}_{\boldsymbol{p}_{, 1}}\left(c_{1 \gamma}+\delta c_{1 \gamma}\right) ; \\
Z \text { coupling : } & \boldsymbol{Z} \sqrt{g_{1}^{2}+g_{2}^{2}}\left(\frac{1}{4}-\frac{2}{3} \sin ^{2} \theta_{W}\right) \boldsymbol{O}_{\boldsymbol{p}_{, 1}}\left(c_{1 Z}+\delta c_{1 Z}\right),
\end{aligned}
$$




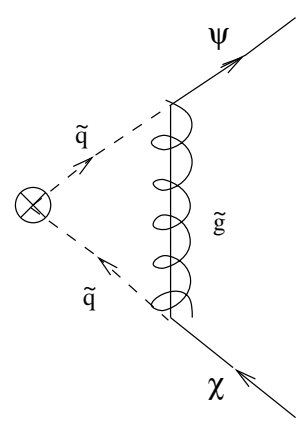

(a)

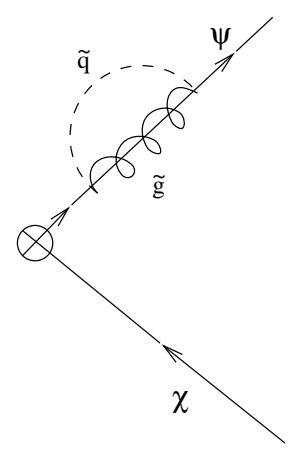

(b)

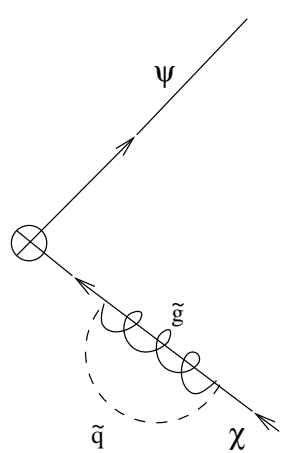

(c)

FIG. 1. One-loop supersymmetric correction of the $t \bar{t}$ pair production current. The arrow shows the flow of quark number and $\tilde{q}$ can be either $\tilde{t}_{1}$ or $\tilde{t}_{2}$.

where at leading order in perturbative QCD: $c_{1 \gamma, Z}=1$. We consider only the contributions from stop $\tilde{t}$ and gluino, assuming that the flavor mixing between generations in the gluino-quark-squark couplings is small. The contributions from $\mathrm{SU}(2)_{L}, \mathrm{U}(1)_{Y}$ gauginos are suppressed by the electroweak couplings. Let $m_{\tilde{t}_{1,2}}$ denote the masses of the two physical stop mass eigenstates, with the mixing angle $\theta$ :

$$
\left(\begin{array}{c}
\tilde{t}_{L} \\
\tilde{t}_{R}
\end{array}\right)=\left(\begin{array}{rr}
\cos \theta & \sin \theta \\
-\sin \theta & \cos \theta
\end{array}\right)\left(\begin{array}{l}
\tilde{t}_{1} \\
\tilde{t}_{2}
\end{array}\right),
$$

and $m_{\tilde{g}}$ denote the gluino mass. The loop corrections to the production current associated with a photon or $Z$ boson give (neglecting terms suppressed by higher powers of $v$ ) :

$$
\begin{aligned}
\delta c_{1 \gamma} & =C_{F} \frac{\alpha_{s}}{4 \pi}\left[F\left(m_{\tilde{t}_{1}}, m_{\tilde{g}}, m_{t}\right)+F\left(m_{\tilde{t}_{2}}, m_{\tilde{g}}, m_{t}\right)+G\left(m_{\tilde{t}_{1}}, m_{\tilde{g}}, m_{t}\right)+G\left(m_{\tilde{t}_{2}}, m_{\tilde{g}}, m_{t}\right)\right] \\
\delta c_{1 Z} & =C_{F} \frac{\alpha_{s}}{4 \pi}\left\{\frac { 1 } { \frac { 1 } { 4 } - \frac { 2 } { 3 } \operatorname { s i n } ^ { 2 } \theta _ { W } } \left[\left(\frac{1}{2} \cos ^{2} \theta-\frac{2}{3} \sin ^{2} \theta_{W}\right) F\left(m_{\tilde{t}_{1}}, m_{\tilde{g}}, m_{t}\right)\right.\right. \\
& \left.\left.+\left(\frac{1}{2} \sin ^{2} \theta-\frac{2}{3} \sin ^{2} \theta_{W}\right) F\left(m_{\tilde{t}_{2}}, m_{\tilde{g}}, m_{t}\right)\right]+G\left(m_{\tilde{t}_{1}}, m_{\tilde{g}}, m_{t}\right)+G\left(m_{\tilde{t}_{2}}, m_{\tilde{g}}, m_{t}\right)\right\}
\end{aligned}
$$

where

$$
\begin{aligned}
F\left(m_{\tilde{t}_{i}}, m_{\tilde{g}}, m_{t}\right) & =\int_{0}^{1} \mathrm{~d} x\left[1-x+\frac{m_{\tilde{t}_{i}}^{2}+\left(x^{2}-1\right) m_{t}^{2}}{m_{\tilde{t}_{i}}^{2}-m_{\tilde{g}}^{2}-m_{t}^{2}} \ln \frac{x m_{\tilde{t}_{i}}^{2}+(1-x) m_{\tilde{g}}^{2}+\left(x^{2}-x\right) m_{t}^{2}}{m_{\tilde{t}_{i}}^{2}+\left(x^{2}-1\right) m_{t}^{2}}\right. \\
& \left.+x \ln \frac{x m_{\tilde{t}_{i}}^{2}+(1-x) m_{\tilde{g}}^{2}-x(1-x) m_{t}^{2}}{(1-x) m_{\tilde{t}_{i}}^{2}+x m_{\tilde{g}}^{2}-x(1-x) m_{t}^{2}}\right] ; \\
G\left(m_{\tilde{t}_{i}}, m_{\tilde{g}}, m_{t}\right) & =\int_{0}^{1} \mathrm{~d} x \frac{-2 x(1-x)\left(x m_{t}^{2} \pm m_{\tilde{g}} m_{t} \sin 2 \theta\right)}{x m_{\tilde{t}_{i}}^{2}+(1-x) m_{\tilde{g}}^{2}-x(1-x) m_{t}^{2}} ; \quad+\text { for } i=1, \quad-\text { for } i=2 ;
\end{aligned}
$$

and $C_{F}=4 / 3$ for $\mathrm{SU}(3)_{c}$.

In realistic SUSY models, for the squark mass matrix in the $\left(\tilde{q}_{L}, \tilde{q}_{R}\right)$ basis, the diagonal matrix elements $M_{L L, R R}^{2} \sim M_{\mathrm{SUSY}}^{2}$, while the off-diagonal matrix element $M_{L R}^{2} \sim M_{\mathrm{SUSY}} m_{t}$. We chose the mass parameters randomly in the intervals: 


$$
\begin{aligned}
(200 \mathrm{GeV})^{2} & \leq M_{L L, R R}^{2} \leq(1000 \mathrm{GeV})^{2}, \\
-(1000 \mathrm{GeV}) m_{t} & \leq M_{L R}^{2} \leq(1000 \mathrm{GeV}) m_{t}, \\
200 \mathrm{GeV} & \leq\left|m_{\tilde{g}}\right| \leq 1000 \mathrm{GeV},
\end{aligned}
$$

with the requirement that the physical stop masses stay within the range of $200 \mathrm{GeV} \leq$ $m_{\tilde{t}_{1}}<m_{\tilde{t}_{2}} \leq 1000 \mathrm{GeV}$. The numerical results for the SUSY correction to the Wilson coefficient $c_{1}$ is shown in Fig. 2 for the currents associated with a photon [(a), (b) and (c)] and a $Z$ boson [(d), (e) and (f)], respectively. The corrections relative to the tree level value are small in both cases: $-0.2 \%<\delta c_{1 \gamma}<0.3 \%$ with $97 \%$ of the points falling between $\pm 0.1 \%$ for the photon, and $-0.6 \%<\delta c_{1 Z}<0.6 \%$ with $86 \%$ of the points falling between $\pm 0.1 \%$ for the $Z$. The enhancement in the correction to the $Z$ current is due to the mixing between the $\tilde{t}_{L}$ and $\tilde{t}_{R}$, which alleviates the partial cancellation between the tree level left and right handed $Z$-current.

$\delta c_{1}$ goes to zero for large squark and gluino masses, since the SUSY contributions decouple for heavy super particles. However, the gluino decouples slower, with $\delta c_{1} \sim m_{t} /\left|m_{\tilde{g}}\right|$ [unlike $\delta c_{1} \sim\left(m_{t} / m_{\tilde{q}}\right)^{2}$ for the squark], as can be seen in $G\left(m_{\tilde{t}_{i}}, m_{\tilde{g}}, m_{t}\right)$ in Eq. (13)). The largest contribution comes from the term proportional to $\sin 2 \theta$. For the $Z$ current, the contribution from the other terms are enhanced due to the left-right mixing in the stop sector, which causes the shift in the $\theta$ dependence in Fig. 2(f).

The SUSY corrections are small compared with the $\mathcal{O}\left(\alpha_{s}^{2}\right)$ NNLL SM contribution to $c_{1}$ [9]. They are also smaller than the theoretical uncertainties in SM predictions at NNLL level [2], which are about $2-3 \%$ due to the variation in $\nu$, for soft and ultrasoft renormalization scales $\mu_{S}=m_{t} \nu$ and $\mu_{U S}=m_{t} \nu^{2}$. Therefore, the corrections caused by the existence of heavy supersymmetric particles are negligible and it is sufficient to extract the top quark mass, decay width and top Yukawa coupling from the $t \bar{t}$ pair production near threshold based on SM calculations.

In summary, we calculated the leading SUSY contribution to top-antitop threshold production via its corrections to the matching condition for the potential and the Wilson coefficient $c_{1}$ of the ${ }^{3} S_{1}$ production current associated with a photon or $Z$ boson using the NRQCD framework. The correction to the potential is zero (at leading order in $v$ ) due to $\mathrm{SU}(3)_{c}$ gauge invariance, which is true in general for any new physics that enters above the electroweak scale. The shape of the top pair production cross section near threshold is unaffected, which can therefore lead to a precise determination of the top quark mass based on the SM calculations. The correction to the coefficient $c_{1}$ of the production current is within $\pm 0.1 \%$ in most cases, which is smaller than the SM NNLL corrections and the theoretical uncertainties from higher orders in QCD perturbation theory.

We have only considered the leading SUSY contribution from the gluino and stop, neglecting terms proportional to higher powers of $\alpha_{s}$ and $v$. Flavor mixing between generations is usually small and not taken into consideration here. Neutralino, chargino and other squarks would contribute through electroweak loops, however their corrections are suppressed by the weak coupling constant instead of strong coupling. If there are super particles lighter than top quark, additional contributions to the running of the quark-antiquark potential and the Wilson coefficient of the production current from $m_{t}$ to lower scales should be taken into account. 

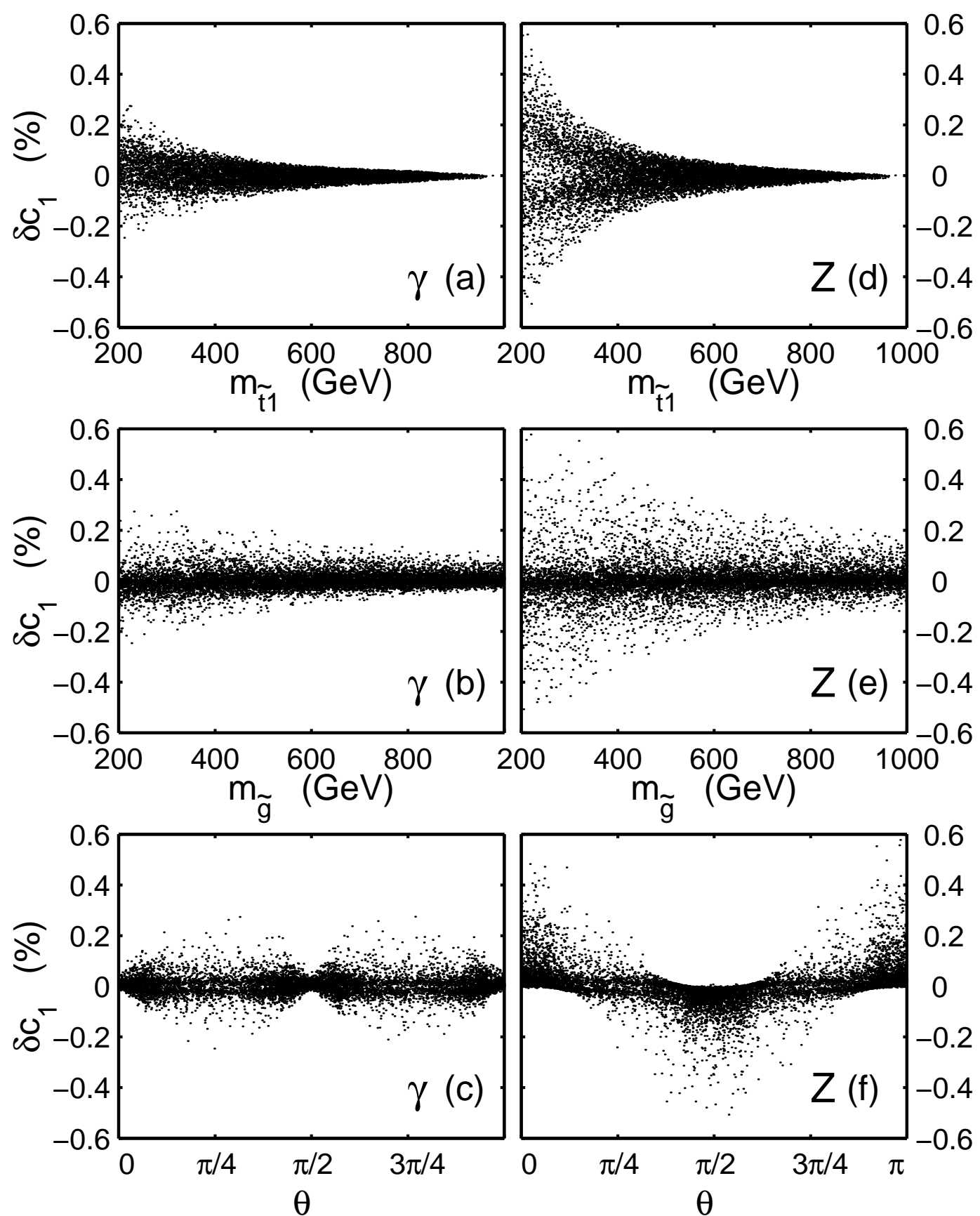

FIG. 2. Scatter plot of SUSY correction to $c_{1}$, for the currents associated with a photon [Fig. (a), (b), (c)] and a $Z$ boson [Fig. (d), (e), (f)]. $m_{\tilde{t}_{1}}$ and $m_{\tilde{g}}$ are the masses of the lighter stop and gluino, respectively, and $\theta$ is the stop mixing angle. The range of the SUSY mass parameters are chosen as described in the paper. 


\section{ACKNOWLEDGMENTS}

We would like to thank I. Stewart for useful discussion on $t \bar{t}$ threshold production. S.S. and M.B.W. are supported by the DOE under grant DE-FG03-92-ER-40701. 


\section{REFERENCES}

[1] A. H. Hoang et. al., Eur. Phys. J. direct C 3, 1 (2000) and references therein.

[2] A. H. Hoang, A. V. Manohar, I. W. Stewart, T. Teubner, Phys. Rev. Lett. 86, 1951 (2001).

[3] G. T. Bodwin, E. Braaten and G. P. Lepage, Phys. Rev. D 51, 1125 (1995).

[4] M. E. Luke, A. V. Manohar and I. Z. Rothstein, Phys. Rev. D 61, 074025 (2000).

[5] W. Hollik, C. Schappacher, Nucl. Phys. B 545, 98 (1999).

[6] J. Guasch, W. Hollik, J. I. Illana, C. Schappacher, J. Sola, hep-ph/0003109 and references therein.

[7] A. V. Manohar, I. W. Stewart, Phys. Rev. D 63, 054004 (2001).

[8] A. V. Manohar, I. W. Stewart, Phys. Rev. D 62, 074015 (2000); Phys. Rev. D 62, 014033 (2000).

[9] A. Czarnecki, K. Melnikov, Phys. Rev. Lett. 80, 2531 (1998); M. Beneke, A. Signer, V. A. Smirnov, Phys. Rev. Lett. 80, 2535 (1998). 